\title{
The use of PIC Microcontroller in Hearing Aids with Automatic Gain Control
}

\author{
Nivin Ghamry \\ Department of Information Technology, \\ Cairo University, Faculty of \\ Computers and Information, \\ Cairo, Egypt
}

\begin{abstract}
PIC Microcontrollers have been achieving low-cost product development and control solutions for medical device manufacturers over the past years. This paper presents a PIC microcontroller-based automatic gain control system for digital hearing aid devices. Noise reduction and speech enhancement are achieved through a wavelet transform-based digital signal processing unit. According to the recognized noise condition in the audio signals, the microcontroller is programmed to send a control signal to the digital volume controller to adjust the volume level in real time. Experimental tests are carried out on real data of hearing impairments suffering from different types of hearing losses. The microcontroller used in this work is the high performance enhanced flash PIC18F4520 of microchip which has an onchip analog to digital converter (ADC) peripheral. The ADC features of PIC are used to detect and compare the sound level. ' $\mathrm{C}$ ' and assembly language programs are developed to control the function of the microcontroller. MPLAB Integrated Development Environment (IDE) is applied for the development of the proposed embedded application employing PIC microcontroller.
\end{abstract}

\section{Keywords}

automatic gain control, hearing aid, PIC microcontrollers, stationary wavelet transform.

\section{INTRODUCTION}

The rapid evolution in electronics is enabling more innovation in medical devices intended for use in variable applications. Microcontrollers and non-volatile EEPROM memories are enabling new medical devices to keep ahead of market trends. PIC microcontrollers achieve low-risk product development by providing seamless program size expansion. Pin compatibility facilitates drop-in replacements of package types as well as variations of reprogrammable (Flash) and one-time programmable (OTP) program memories rather than code re-writing. This seamless migration path allows medical device designers to reuse verified code and a proven printed circuit board layout, potentially reducing the burden of regulatory compliance. In Table 1 some of the medical devices in which PIC microcontrollers were successfully applied are listed. These devices include motors which are controlled by PIC microcontrollers of various families[1].

The Table also illustrates the PIC microcontroller family used and a brief explanation of the function of the microcontroller in each medical device is given.
Table 1. Microcontroller-based medical devices

\begin{tabular}{|c|c|c|}
\hline Medical application & PIC family & Function of PIC \\
\hline $\begin{array}{l}\text { Sphygmomanometer or } \\
\text { blood pressure meter. }\end{array}$ & PIC16F91X & $\begin{array}{l}\text { The cuff inflation } \\
\text { and deflation are } \\
\text { handled by a small } \\
\text { air compressor } \\
\text { driven by an } \\
\text { electric motor }\end{array}$ \\
\hline $\begin{array}{l}\text { Oxygen Concentrator } \\
\text { produces a supply of air } \\
\text { with increased oxygen } \\
\text { content to replace } \\
\text { liquid oxygen or } \\
\text { pressurized oxygen } \\
\text { tanks for people who } \\
\text { require oxygen rich air. }\end{array}$ & $\begin{array}{l}\text { dsPIC30F40 } \\
11\end{array}$ & $\begin{array}{l}\text { The compressor } \\
\text { that moves air into } \\
\text { the oxygen } \\
\text { concentrator is } \\
\text { driven by an } \\
\text { electric motor }\end{array}$ \\
\hline $\begin{array}{l}\text { Pulse oximeter } \\
\text { measures the amount of } \\
\text { oxygen in blood by } \\
\text { sensing the amount of } \\
\text { light absorbed in } \\
\text { capillaries under the } \\
\text { skin using a photodiode }\end{array}$ & PIC24FJ & $\begin{array}{l}\text { The output voltage } \\
\text { of the photodiodes } \\
\text { read by an analog- } \\
\text { to-digital converter } \\
\text { on a PIC which } \\
\text { calculates the ratio } \\
\text { of red light to } \\
\text { infrared light, and } \\
\text { determines the } \\
\text { corresponding } \\
\text { oxygen saturation } \\
\text { level using a } \\
\text { lookup table. }\end{array}$ \\
\hline $\begin{array}{l}\text { Continuous positive } \\
\text { airway pressure } \\
\text { (CPAP) machines } \\
\text { deliver therapy for } \\
\text { obstructive sleep apnea } \\
\text { (OSA) people who } \\
\text { experience a collapse of } \\
\text { the airway during sleep. } \\
\text { It keeps the airway } \\
\text { open, allowing a } \\
\text { normal sleep pattern. }\end{array}$ & dsPIC33 & $\begin{array}{l}\text { CPAP machine } \\
\text { includes a motor to } \\
\text { drive a fan which } \\
\text { pressurizes the air. }\end{array}$ \\
\hline Glucometers determine & MCP3421 & $\begin{array}{lr}\text { The } & \text { catalytic } \\
\text { reaction } & \text { generates }\end{array}$ \\
\hline
\end{tabular}




\begin{tabular}{|c|c|}
\hline $\begin{array}{l}\text { the concentration of } \\
\text { glucose in a blood for } \\
\text { the management of } \\
\text { diabetes through } \\
\text { catalytic reaction }\end{array}$ & $\begin{array}{l}\text { a voltage that can } \\
\text { be measured by the } \\
\text { PIC } \\
\text { microcontrollers' } \\
\text { embedded analog } \\
\text { to-digital converter } \\
\text { (ADC). }\end{array}$ \\
\hline
\end{tabular}

Beside the above mentioned medical devices, this current work focus on the application of microcontrollers in hearing aid devices. In the following a sample of previous research work is illustrated. In [2] the design and implementation of a microcontroller based electronic hearing aid with output level indicator is explored. In[3] and [4] different designs of a microcontroller-based hearing aid device are provided, by which power consumption and hardware area are reduced. However, the use of embedded systems and microcontrollers in hearing aid devices with the feature of automatic gain control (AGC) is still limited and less research work can be found in this field [5]. In this paper a PIC microcontrollerbased automatic gain control system for digital hearing aid devices is presented. According to the recognized noise condition in the audio signals, the microcontroller is programmed to send a control signal to AGC unit to adjust the gain level. The signal processing module is based on wavelet transform for noise reduction and speech enhancement. Experimental results are based on real data of hearing impairments who suffer from different kinds of hearing loss. The simulation results obtained show that using microcontroller-based AGC hearing device could provide an adequate fast response for the user and that the proposed system can track the variations in the input audio signal in real time.

This paper is organized as follows, In section 2 the principles of hearing aid devices with AGC are briefly overviewed. In section 3 the stationary wavelet transform-based DSP unit is discussed. In Section 4 the proposed microcontroller-based architecture is explained. Section 5 presents experimental results based on real data of patients suffering from different types of sensor neural hearing losses(SNHL). Section 6 concludes the presented work.

\section{HEARING AID DEVICES WITH AUTOMATIC GAIN CONTROL SYSTEMS}

\subsection{Automatic gain control in receivers}

Automatic gain control (AGC) circuit is a closedloop feedback regulating circuit [6]. It was implemented in first receivers to maintain a constant signal level at the output, independent of the signal variations at the input of the system, known as fading propagation. Now AGC circuits can be found in any device or system where amplitude variations over a wide dynamic range in the output signal could lead to loss of information or to an unacceptable performance of the system. This requires the design of specific circuits for continuous adjustments in the receiver gain so that circuits following the AGC circuit require less dynamic range. If the changes in the signal level are much slower than the information rate contained in the signal, then an AGC circuit can be used to provide a signal with a well-defined average level to downstream circuits. In several system applications, the time to adjust the gain in response to an input amplitude change should remain constant, independent of the input amplitude level and hence gain setting of the amplifier. The large dynamic range of signals that must be handled by most receivers requires gain adjustment to avoid overload of the stages and to adjust the demodulator input level for optimum operation. Figure 1 illustrates a block diagram of the circuit[7]. As the figure shows the input signal is amplified by a Variable Gain Amplifier (VGA), whose gain is controlled by an external signal. The output of the $\mathrm{VGA} \mathrm{V}_{1}$, is amplified by a second stage to generate an adequate level of $\mathrm{V}_{\mathrm{o}}$. The output signal parameters, such as amplitude, carrier frequency, index of modulation or frequency, are sensed by the detector and any undesired component is filtered out. The remaining signal is compared with a reference signal $V_{R}$. The result of the comparison is used to generate the control voltage $\mathrm{V}_{\mathrm{c}}$ and adjust the gain of the VGA.

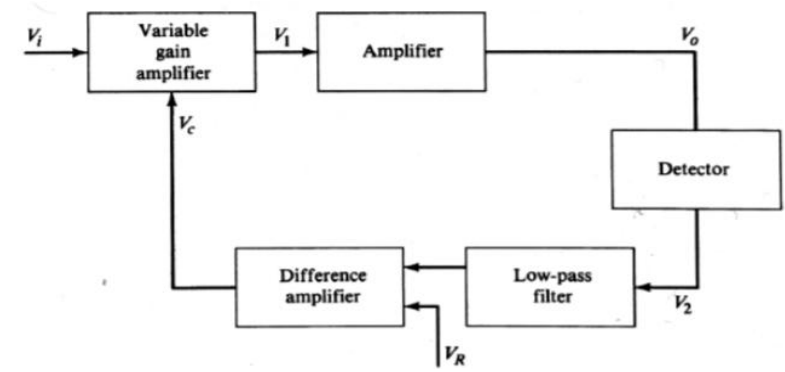

Fig 1: Block diagram of AGC.

An AGC circuit in the receiver provides a substantially constant signal level to the demodulator independent of the input signal level. The AGC ideal transfer function is shown in Figure 2 [7]. For low input signals the AGC is disabled and the output is a linear function of the input, when the output reaches a threshold value $\left(\mathrm{V}_{1}\right)$ the AGC becomes operative and maintains a constant output level until it reaches a second threshold value $\left(\mathrm{V}_{2}\right)$. At this point, the AGC becomes inoperative again, this is usually done in order to prevent stability problems at high levels of gain.

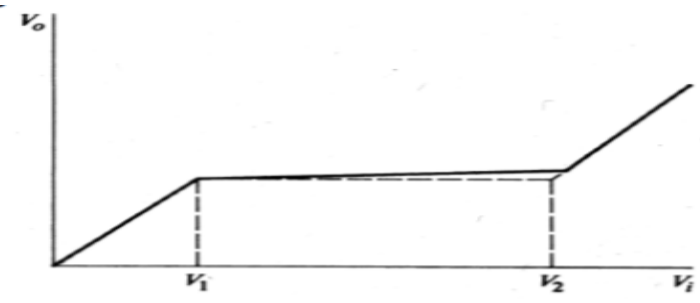

Fig 2: AGC ideal transfer function. 


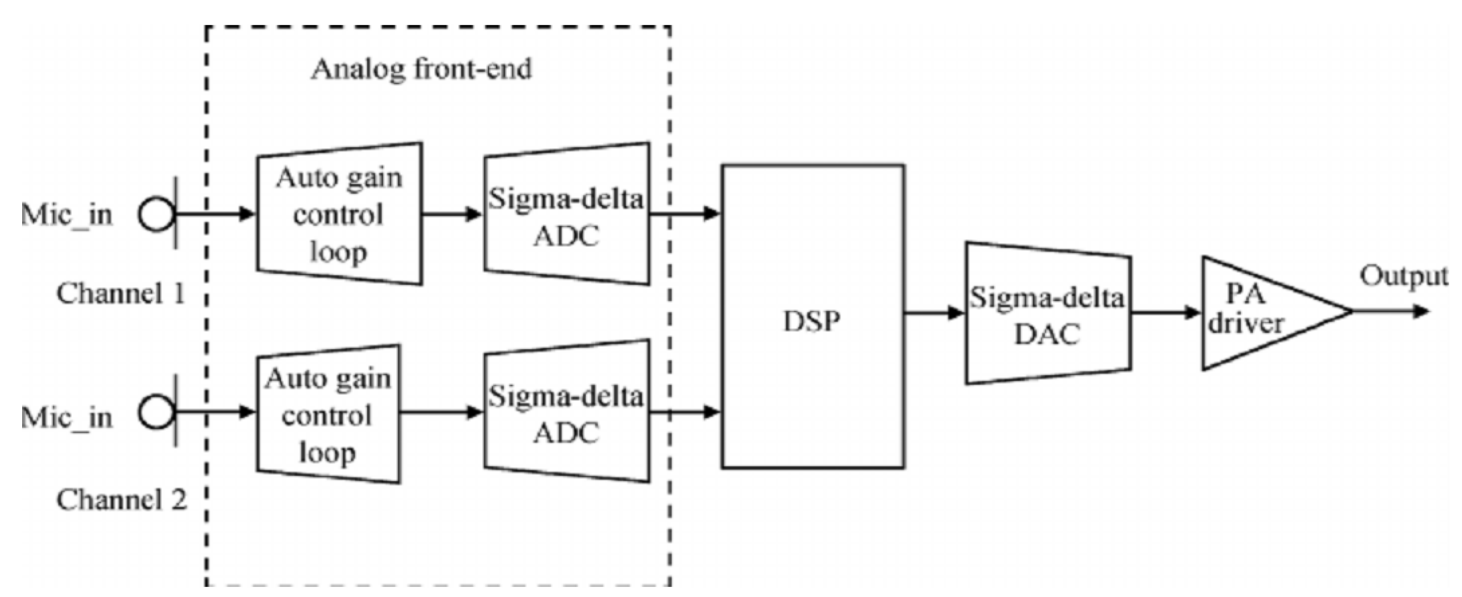

Figure 3. A two-channel digital hearing aid system

\subsection{AGC in hearing aids}

Former research has revealed the degrees of hearing loss as follows[8]

1. Normal hearing $(0-25 \mathrm{~dB})$

2. Mild hearing loss $(26-40 \mathrm{~dB})$ causes inattention, difficulty in suppressing background noise, and increased listening efforts.

3. Moderate hearing loss $(41-55 \mathrm{~dB})$ affects language development, syntax and articulation.

4. Moderate to severe hearing loss $(56-70 \mathrm{~dB})$ causes decreased speech intelligibility.

5. Severe hearing loss (71-90 dB) affects voice quality.

6. Profound hearing loss ( $>90 \mathrm{~dB})$ (deafness) by which speech and language deteriorate.

For most patients with hearing loss, simply increasing or decreasing the volume does not improve the clarity of speech. They might need decreasing sounds that are too loud, and increasing other sounds to improve speech understanding. The automatic gain control (AGC)feature is found in most modern hearing aids[9]. The goal of variable gain amplification is to make soft sounds audible, moderate sounds comfortable and clear, and loud sounds tolerable. Recently, hearing aids separate sound into different frequency (tonal) regions, called channels. The volume of each channel can be adjusted independently, allowing for certain sounds to be amplified more than others. The amount of amplification in each channel is adjusted using the hearing aid programming software. The number of channels available for programming differs among hearing aids. With more channels, hearing aids can be programmed to make the analysis of the sound environment more accurate. However, more than 15 to 20 channels can cause sounds to become 'muddy'. The AGC also controls the levels of sound in relation to feedback suppression, noise reduction, speech enhancement, and output limiting. Figure 3. shows a two-channel digital hearing aid system. It consists of five blocks: an auto- gain control loop (AGC), a sigma-delta analog-to-digital converter (SDADC), a digital signal processor (DSP), a sigma- delta digital-to-analog converter (SDDAC) and a power amplifier driver. To ensure normal reception of the human ear, the AGC gain must involve a wide range of changes, as follows:
1. when the sound level is in the 50 to $80 \mathrm{~dB}$ SPL range, the AGC needs to provide $0-30 \mathrm{~dB}$ gain.

2. when the sound level is sufficiently large enough at about 80 or $90 \mathrm{~dB}$ SPL, the gain of the AGC is zero.

3. when the sound level is above $90 \mathrm{~dB}$ SPL, which is a dangerous signal level for people, the AGC should provide proper attenuation of gain to protect the ears.

\section{THE WAVELET TRANSFORM- BASED DSP UNIT}

In this work a signal processing core based on stationary wavelet packets is applied for denoising and speech enhancement. The denoising method exploits the features of the Daubechies4 wavelet packet analysis in the stationary wavelet basis domain for decomposition[10]. To get the optimum representation of the decomposed signal, the best tree selection algorithm is applied on the non-decimated stationary wavelet coefficients. Denoising of the coefficients is achieved via soft thresholding given by Eq.(1)

$$
T_{j}=\sigma_{j} \sqrt{2 \ln \frac{N}{2^{j}}}
$$

Where $\mathrm{N}$ is the length of the input signal and $\sigma_{j}$ is the standard deviation of noise at scale j. $T_{j}$ is the threshold calculated on a level-dependent basis[11]. According to wavelet decomposition, each of the wavelet coefficients corresponds to a band of frequencies. WPT-based speech enhancement is achieved by frequency dependent amplification. The amplification gain is calculated separately for each wavelet coefficient based on its intensity level according to the time varying frequency-dependent processing (TVFD) gain calculation [12]. The gain is calculated such that the ratio of log intensity above hearing is the same for the hearing-impaired listener as the corresponding ratio is for the normal-hearing listener. This is given mathematically by Eq. (2):

$$
\frac{\delta^{*}}{\delta}=\frac{\Delta^{*}}{\Delta}
$$

Where $\delta$ is the distance between the unamplified wavelet coefficient and the normal hearing threshold. $\Delta$ is the dynamic range of a normal-hearing person (in $\mathrm{dB}$ ), $\Delta^{*}$ is the dynamic 


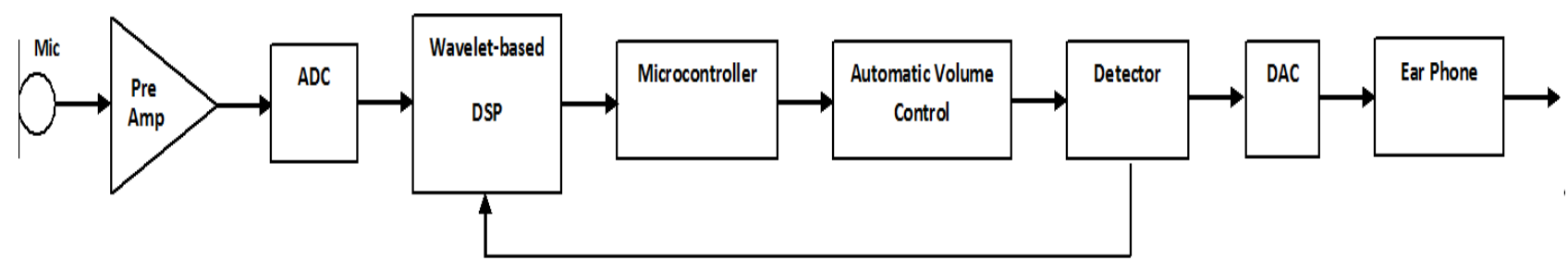

Fig.4 Block diagram of the microcontroller-based AGC architecture.

range of a hearing- impaired person (in $\mathrm{dB}$ ). From the above relationship, compression gain for a coefficient can be derived using logarithmic representations in Eq.(3):

$$
C^{*}=T_{i m p}+\left(C-T_{\text {norm }}\right) \frac{\Delta^{*}}{\Delta}
$$

Where $T_{i m p}$ is the threshold of hearing of a hearing-impaired person (in $\mathrm{dB}$ ), $T_{\text {norm }}$ is the threshold of hearing of a normal person (in $\mathrm{dB}$ ). $C^{*}$ and $C$ are the compensated and noncompensated wavelet coefficients, respectively. For sounds of small Sound Pressure Level (SPL) the gain is adjusted for overcoming the loss of sensitivity along the whole frequency range according to the different levels. The value of the gain can be raised at certain frequencies to smooth the transition from mild- to severe hearing loss. For sounds of larger SPL, the gain is adjusted at high-frequencies according to the loudness growth function to avoid discomfort caused by the reduced dynamic range of the listener. The speech signal is reconstructed from the modified coefficients after applying amplification gain.

\section{MICROCONTROLLER BASED ARCHITECTURE FOR AGC}

The Microcontroller section forms the control unit of the hearing aid device. It basically consists of the PIC microcontroller with its associated circuitry like crystal with capacitors, reset circuitry and pull up resistors. Each type of PIC microcontroller provides a set of different of features, thus the most suitable microcontroller can be selected for any given application. Some of the main selection criteria are: number of I/O pins available, program memory type and size, timers, interrupt sources, analog inputs (8-bit or 10-bit), serial communication interfaces (USART, SPI, I2C, CAN), internal oscillator, in-circuit debugging, etc. When developing an embedded system, the number and type of inputs and outputs need to be determined. After the hardware requirements have been established, the program is written and tested. According to the size of the program, the chip memory size can be determined.

\subsection{The Microcontroller Module}

In this work, the powerful PIC18F4520 8-bit microcontroller of microchip is used. It is specially chosen because of several features making it ideal for advanced level analog to digital applications in the various fields. These features include CMOS technology, low power, high speed FLASH/EEPROM, fully static design, wide operating voltage range $(2.0 \mathrm{~V}$ to $5.5 \mathrm{~V})$, industrial and extended temperature ranges. According to [13] PIC18F4520 microcontroller forty pins, of which seven are important non I/O pins. These pins include the MCLR (master clear) pin, which is active low. A switch is connected from that pin to ground to reset PIC when necessary. Biasing are Vdd (pin 11 and 32) and Vss (pin 12, 31 ). An oscillator is connected across pin 13 and 14 to provide external clock and the timing signals necessary for program execution. The remaining 33 pin are configured as I/O pin. The analog signal received is interfaced with the ADC peripheral of the PIC microcontroller where it is converted to a digital outputs.

\subsection{The ADC Module}

The ADC module on the PIC has four special function registers associated with it: Result High Register (high byte) ADRESH , Result Low Register (low byte) ADRESL to store the output from the converter, Control Register 0 (ADCON0) and Control Register 1 (ADCON1). The ADCONO register is used to set the conversion time and select the analog input channel. The ADON bit is used to turn on the ADC else the $\mathrm{ADC}$ is turned off when the microcontroller is powered up to reduce power consumption. ADCS1 and ADCS0 set the conversion time. The GO_DONE bit is used to check if the conversion is finished. Setting this bit initiates the start of conversion then the bit is cleared when the conversion is complete. CHS2, CHS1 and CHS0 are the channel select bits to determine which input pin is routed to the ADC. ADCON1 is split into two sections. The first section is a single bit, the result format selection bit ADFM which selects if the output is right justified (bit set) or left justified (bit cleared). The advantage is the possibility to use as an 8 bit converter (instead of ten bit) by clearing this bit, and reading just ADRESH and ignoring the two least significant bits in ADRESL. The second section includes the A/D port configuration control bits PCFG3-0. The default of PCFG = 0000 makes the 8 pins RA0-RA3 and RA5 as well as RE0RE2 used for analog inputs. The internal RC oscillator is used for the conversion clock source.

\subsection{The Digital to Analog Converter}

The DAC MCP4921 applied in this work belongs to the Microchip Technology Inc. MCP492X. MCP492X are $2.7-$ $5.5 \mathrm{~V}$, low-power 12-Bit DACs with Serial Peripheral Interface (SPI) which provides high accuracy and low noise performance for applications where calibration or compensation of signals is required. SPI is a standard for 


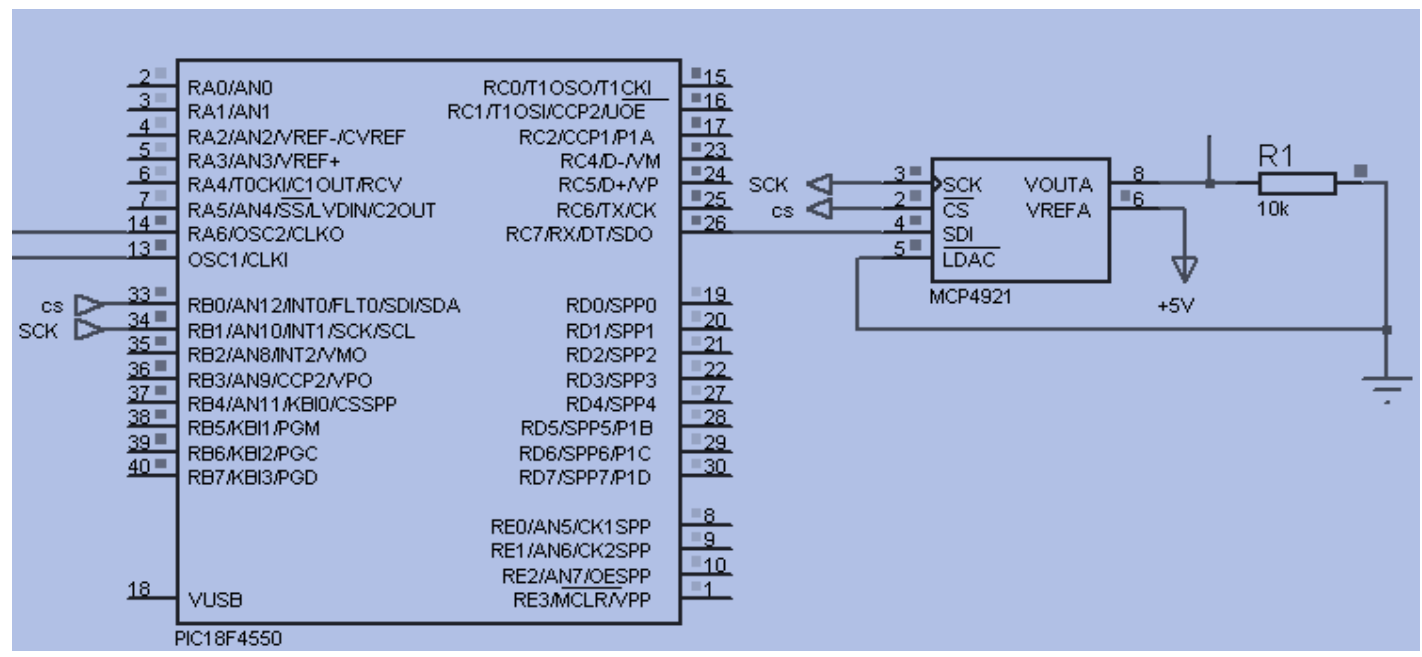

Fig. 5 Schematic diagram of the PIC microcontroller and the DAC MCP4921.

Controlling almost any digital electronics that accepts a clocked serial stream of bits. MCP4921 is an easy-to- program and configure DAC as it only accepts a 2 byte instruction of which 12 bits is the data and the 4 most significant bits are used to control the device. More about MCP4921 is found in [1]. Fig.5 shows the schematic diagram of the PIC microcontroller and the DAC MCP4921.

\section{EXPERIMENTAL RESULTS}

In the following the experimental results of Pure Tone Audiometry (PTA) hearing test are illustrated. PTA is the basis for diagnosis identifying hearing threshold levels. It enables determination of the degree, type and configuration of a hearing loss. PTA is a subjective, behavioral measurement of hearing threshold, as it relies on patient response to pure tone stimuli. PTA provides ear specific thresholds, and uses frequency specific pure tones to give place specific responses, so that the configuration of a hearing loss can be identified. Hearing sensitivity is plotted on an audiogram, which is a graph displaying intensity as a function of frequency. The following types of hearing loss are displayed on PTA[14]. Firstly, conductive hearing loss, which is secondary to an outer ear or middle ear abnormality. It reduces the effective intensity of the air-conducted (AC) signal reaching the cochlea, but it does not affect the boneconducted (BC) signal. AC thresholds are poorer than normal by at least $10 \mathrm{~dB}$. Secondly, sensor neural hearing loss has AC and $\mathrm{BC}$ thresholds within $10 \mathrm{~dB}$ of each other, and thresholds are higher than $25 \mathrm{~dB}$ HL. Thirdly, mixed hearing loss has conductive and sensor neural components. Fig.6 shows a chart of hearing sensitivity versus frequency. For threshold testing intensity, decibels are measured in hearing level (HL), which is based on the standardized average of individuals with normal hearing sensitivity. Usually frequencies of 250-8000 $\mathrm{Hz}$ are used in testing because this range represents most of the speech spectrum. Fig.6 shows the PTA of a hearing impairment suffering of bilateral moderate to severe SNHL, having $\mathrm{AC}$ of $58 \mathrm{~dB}$ marked in the figure with ' $\mathrm{X}$ ', and $\mathrm{BC}$ of $50 \mathrm{~dB}$ marked in the figure with ' $\mathrm{O}$ ' in the right and left ear.

The aided response, marked in the figure with 'A', using a non AGC hearing aid is not adequate.

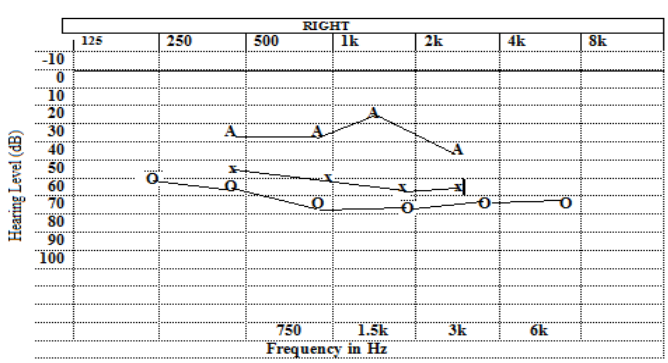

(a)

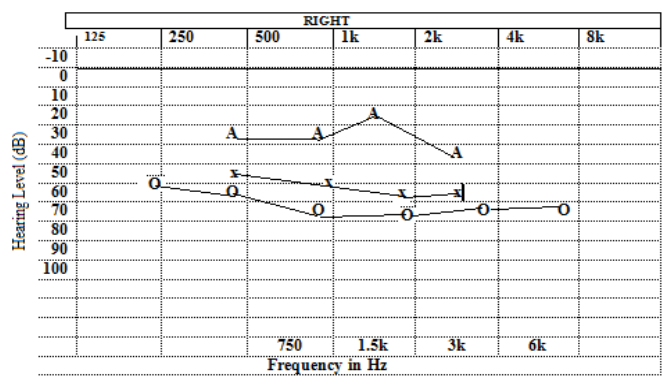

(b)

Fig 6 : PTA of inadequate aided response

Fig.7 shows PTA of another patient of normal hearing on the right ear, having $\mathrm{AC}$ of $21 \mathrm{~dB}$ marked in the figure with ' $\mathrm{X}$ ', and $\mathrm{BC}$ of $20 \mathrm{~dB}$ marked in the figure with 'O'. On the left ear the patient suffers from severe mixed SNHL. AC is $90 \mathrm{~dB}$ marked in the figure with ' $\mathrm{X}$ ' and $\mathrm{BC}$ is $40 \mathrm{~dB}$ marked in the figure with ' $\mathrm{O}$ '. The figure shows that the AGC hearing aid could provide a very good response in the left ear.

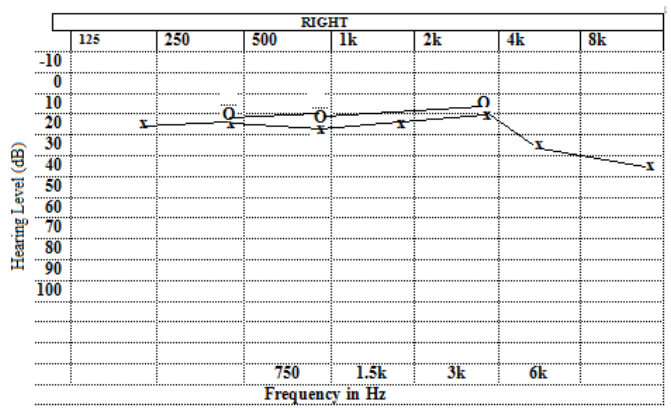

(a) 


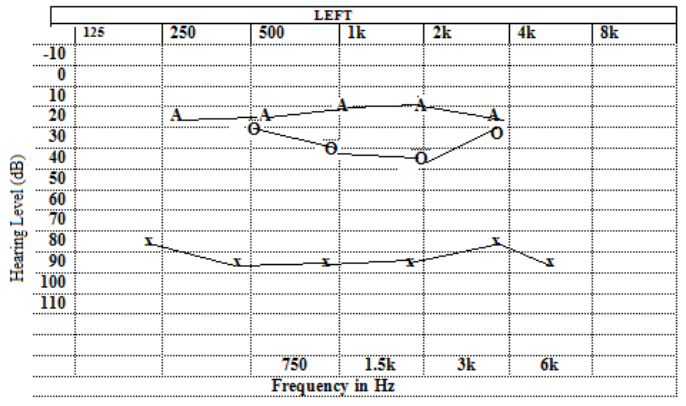

(b)

Fig 7 : PTA of adequate aided response.

\section{CONCLUSION}

In this paper a PIC18F4520 microcontroller-based automatic gain control system for digital hearing aid devices is presented. The signal processing unit applies stationary wavelet packet algorithm for noise reduction in the audio signal. According to the recognized noise condition in the audio signals, the microcontroller is programmed to send a control signal to the digital volume controller to adjust the volume level in real time. Experimental tests are carried out on real data of hearing impairments suffering from different types of hearing loss. The obtained results show that the proposed AGC hearing aid provides adequate response and complies with the characteristics required by hearing impairment like portability, intelligibility, low price and good performance.

\section{REFERENCES}

[1] www.microchip.com/medical/Soulutions for medical applications

[2] A.O. Eze, G. Ihekweaba and N. R. Chinyere, "Exploring a microcontroller based hearing aid with an output level indicator," International Journal of Computational Engineering Research, vol. 2, pp.253-255.

[3] M. A.A. Mashud, M. Bishwas, S. R.Paul, S. C. Barman and Md. S. Islam, " Microcontroller based low-power consumption smart hearing aid," International Journal of Emerging Trends in Engineering and Development, vol.2, pp. 567-572, Mar. 2013.

[4] N. Ghamry, "A Microcontroller-Based Design for Digital Hearing Aid Devices", International Journal of Emerging Trends in Engineering and Development, vol.4, pp. 407-415, July. 2013

[5] M. Ziad, S. Irtiza and A. Shah, "Audio signal's test in designing a cost-effective hearing aid device using a microcontroller", International Journal of Biomedical Engineering and Technology, vol. 20, pp. 195-207, March 2016

[6] J. Smith; "Modern Communication Circuits"; McGrawHill, 1986.

[7] D. Green; "Global stability analysis of automatic gain control circuits"; Circuits and Systems, IEEE Transactions on; Vol. 30, Issue 2, pp. 78-83, Feb. 1983.

[8] Textbook of Hearing Aid Amplification: Technical and Clinical Considerations. (2000). Publication of Cengage Learning., Robert E. Sandlin.

[9] U. Cheng and S-Yuh Lee and An-Po Lin "A 0.6-V 1.8$\mu \mathrm{W}$ automatic gain control circuit for digital hearing aid", IEEE Asia Pacific Conference on Circuits and Systems, 2008. APCCAS 2008.

[10] N. Ghamry, "FPGA Implementation of Hearing Aids using Stationary Wavelet-Packets for Denoising", International Journal of Computer Applications , Vol.69- No.15,pp. 30-36, May 2013.

[11] M. Lavielle, "Detection of multiple changes in a sequence of dependent variables, Stochastic Processes and their Applications ",Elsevier Science83 (1999) 79102.

[12] L. A., Drake, J. C. Rutledge, and J. Cohen, "Wavelet analysis in recruitment of loudness compensation", IEEE Transaction on Signal Processing, vol.41, pp.3306 $-3312,1993$

[13] British Society of Audiology (2011), Pure tone air and bone conduction threshold audiometry with and without masking and determination of uncomfortable loudness levels. 